REVISTA DEL POSGRADO EN DERECHO DE LA UNAM | revistaderecho.posgrado.unam.mx AÑO 7, No 12, ENERO - JUNIO 2O2O | https://doi.org/10.22201/fder.26831783e.2020.12.140

\title{
HACIA UNA PROPUESTA NORMATIVA CLIMÁTICA CON VISIÓN NICARAGÜENSE
}

Towards a climate regulatory proposal With a NicaRAGUAN VISION

Mario TorRes Lezama ${ }^{1}$

Resumen: El presente artículo ofrece un analísis de la relación de los Derechos Humanos de la tercera generación con el calentamiento global en la busqueda del desarrollo humano sostenible, que luego evalua la situación de vulnerabilidad que posee Nicaragua para brindar una propuesta normativa frente a la amenaza y los impactos del paradigma climático.

Palabras Glave: Derechos Humanos, Sistema Climático, Medio Ambiente, Cambio Climático, Gobernanza, Vulnerabilidad, Mitigación, Adaptación, Gestión Ambiental.

Abstract: This article provides an analysis of the relationship of the Human Rights of the third generation with global warming in the search for sustainable human development, which then assesses Nicaragua's vulnerability situation to provide a normative proposal against the threat and impacts of the climate paradigm.

Keywords: Human Rights, Climate System, Environment, Climate Change, Governance, Vulnerability, Mitigation, Adaptation, Environmental Management.

Sumario: I. Introducción; II. La exigencia del medio ambiente sano como parte de los Derechos Humanos; III. El sistema climático y su vínculo con el Derecho Humano de un medio ambiente sano; IV. La situación nicaragüense frente al cambio climático; V. La vulnerabilidad, la gobernanza ambiental y la gestión climática nicaragüense; VI. Hacia una propuesta normativa climática para enfrentar la problemática; VII. Conclusiones; VIII. Fuentes consultadas.

${ }^{1}$ Doctor en Derecho. Docente e Investigador horario de la Universidad Centroamericana -UCA- Managua, Nicaragua. <Lezatomar@gmail.com>. ORCID: <https://orcid.org/0000-0001-6452-3773>.

Fecha de recepción: 17 de enero de 2020; fecha de aprobación: 16 de abril de 2020. 


\section{INTRODUCCIÓN}

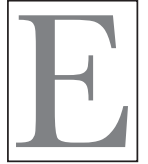

1 cambio climático es un paradigma global. Dicha problemática se manifiesta actualmente con varias complejidades que afectan transversalmente al Derecho Internacional Ambiental moderno tales como: la gobernanza mundial del medio ambiente, la equidad intergeneracional, los Derechos Humanos y la estabilidad de las naciones, entre otras, para disfrutar de un medio ambiente sano, capaz de satisfacer las necesidades de las generaciones actuales y futuras. Es decir, en términos más amplios lo que está en el centro de la discusión es la supervivencia del concepto del desarrollo humano sustentable.

De ahí que, la constante voracidad sobre los recursos naturales y la actitud depredadora contra el medio ambiente, originan básicamente el problema ambiental climático, siendo una consecuencia del modelo de crecimiento económico irracional, del incremento demográfico, del modelo productivo y del consumo ilimitado, del mercado como tal, como valores de la sociedad que resultan insostenibles para continuar de esa manera.

Visto de esa forma, el fenómeno no es solamente un problema ambiental. En realidad, sus consecuencias negativas pueden ser tan complejas y disímiles que pueden afectar en distinta medida en todas las actividades relevantes del género humano, entre las principales: la salud, el acceso a las fuentes de agua, la seguridad alimentaria, el uso de la energía, la protección de la biodiversidad, la economía e incluso la propia existencia de algunos países como las naciones insulares.

Adicionalmente, los países más expuestos a dichas consecuencias serían aquellos que carecen de los recursos y poseen una limitada preparación adecuada, para adaptarse a los cambios venideros. Dicho de otra manera, hablamos de los países en vías de desarrollo (como el caso de Nicaragua), quienes, a pesar de no ser los responsables históricos, deberán asumir los efectos negativos climáticos con mayor crudeza. 
Al mismo tiempo y a diferencia de otros problemas ambientales aparentemente similares, tales como: el agotamiento de la capa de ozono o la lluvia ácida, la pérdida de los ecosistemas de la biodiversidad, deforestación o de la sequía, no existe una única solución técnica contra el cambio climático, para proteger el bien jurídico público conocido como atmósfera, complicando el análisis y la decisión acerca de las medidas e instrumentos normativos más apropiados y la estimación precisa en los costos de las soluciones.

Cabe mencionar que, aunque los cuestionamientos y dudas en materia científica sobre el calentamiento global son siempre bienvenidos, la falta de total certeza en cuanto a la extensión o seriedad de las consecuencias del fenómeno, no significa la ausencia de razones normativas para actuar desde ya, puesto si bien se reconoce que la ciencia del cambio climático dista mucho de ser perfecta, los riesgos, la amenaza y su posibles efectos que prevé la información generada, justifican plenamente la actuación de la comunidad internacional, así como la de los Estados a través de sus autoridades a escala local, para consensuar e implementar medidas de mitigación y adaptación, desde el nivel territorial a un alcance nacional.

$\mathrm{Al}$ respecto Juste Ruiz² afirmó que la comunidad internacional ha optado por la búsqueda de acuerdos e implementación de soluciones en el contexto de un régimen jurídico global y ha perseverado con dicha alternativa, desde la óptica multilateral para enfrentarse al fenómeno climático.

Sin embargo, hoy es posible sostener con más fuerza que hace algunos años, que el actual régimen internacional no ha sido capaz de adoptar medidas de mitigación apropiadas contra las consecuencias negativas del fenómeno del calentamiento global y menos facilitar la adaptación a ésas. De esa manera, se presenta un anteproyecto de Ley para enfrentarse al cambio climático, con una aplicada visión nicaragüense, analizando las condiciones, políticas y estrategias que cuenta dicho país para enfrentarse a dicha problemática global.

${ }^{2}$ Juste Ruiz, José, La Protección del Medio Ambiente en su Dimensión Internacional, Madrid, Mcgraw-Hill/Interamericana de España, 1999. 
II. LA EXIGencia del Medio Ambiente sano como PARTE de los Derechos Humanos

Visto así, la actuación del ser humano debe ser regulada por el Derecho Ambiental, a como lo afirma $\mathrm{Mateo}^{3}$, porque desde la concepción del ser humano, a éste se le otorgan derechos y deberes que deben ser aceptados, tutelados, cuidados y respetados por todas las naciones sin distinción alguna. Por consiguiente, ese conjunto de derechos aglutinados doctrinalmente en los Derechos Humanos es reconocido como atributos, libertades y prerrogativas, que se les otorga a todos los seres de la raza humana y son indispensables para el reconocimiento de una vida digna, los que poseen características tales como: objetivos, imprescriptibles, intransferibles, atemporales, indivisibles y personales que marcan su diferencia frente a otros elementos.

De ahí que, los derechos fundamentales de las personas han adquirido en los últimos años una importancia trascendental nacional e internacionalmente, que se observa con la incorporación en los ordenamientos jurídicos de los Estados del actual sistema internacional, en las políticas de los gobiernos y en el marco jurídico de muchos pueblos, para garantizar los Derechos Humanos de todas las personas, tal a como lo afirma Risse ${ }^{4}$.

Así visto, la historia marca su origen en la edificación de esos derechos con la Declaración de Independencia de los Estados Unidos de América (1776) de la corona inglesa, con la noción jurídica moderna de la igualdad de los hombres, derecho a la justicia, libertad de culto, libertad de prensa, separación de poderes, entre otros. Luego se fortalece esa noción con la Revolución Francesa y la Declaración de los Derechos del Hombre y del Ciudadano de 1789,

\footnotetext{
${ }^{3}$ Martín Mateos, Tratado De Derecho Ambiental, Vol. I, Madrid, Trívium, 1991.

${ }^{4}$ Risse, Thomas, et al., The Power of Human Rights: International Norms and Domestic Change, New York, Cambridge University Press, 1999.
} 
tal a como lo explica Cecchetto ${ }^{5}$.

Es decir, que esos derechos tienen su origen en las proclamas expresadas por la trasformación social revolucionaria, como derechos individuales, políticos y civiles que exigen el respeto a la dignidad de la persona frente a los abusos del poder por el Estado y para imponerle a este último; la abstención o la inhibición de perjudicar o limitar los derechos a la libertad de los ciudadanos como primera generación en esa etapa histórica.

Esa primera generación sustenta los derechos de corte político y social, brindando a las personas la conquista de la libertad, la cual junto con el resultado de las atrocidades durante los conflictos mundiales (Primera y Segunda Guerra Mundial), se vio también complementada su configuración con el nacimiento de la Organización de las Naciones Unidas (ONU por sus siglas) en 1945 y que posteriormente en 1948, se garantiza la tutela de esos derechos con la Declaración Universal de los Derechos Humanos, como parte del proceso de conquista paulatina de todos los derechos de las personas.

Dicho instrumento es considerado como el primer documento de carácter internacional, proclamando los derechos para todas las personas, sin perjuicio de su cultura, nacionalidad, religión, raza, credo o situación económica, a como lo afirmó Jiménez Solares ${ }^{6}$. En esa circunstancia, la guerra fría entre los bloques político/económicos, encabezados por los Estados Unidos y la antigua Unión Soviética, deviene un retroceso en la aplicación de los Derechos Humanos, por el rechazo a la aplicación de la Declaración, tal a

${ }^{5}$ Cecchetto, Sergio, “¿Una Ética de Cara Al Futuro? Derechos Humanos y Responsabilidades de la Generación Presente Frente a las Generaciones por Venir”, Andamios, Revista de Investigación Social, Vol. 3, Núm. 6, 2007, p. 2. Http://Wwre.Redalyc.Org/Articulo. Oa? Id =62830603.

${ }^{6}$ Jimenez Solares, Elba, "Las normas internacionales de derechos humanos y su contribución al orden público internacional", Revista de Derecho, Núm. 14, México, Universidad Nacional Autónoma de México, 2014, pp. 325-347. Https://Dialnet.Unirioja.Es/Servlet/Articulo? Codigo $_{0}=4965197$. 
como lo señaló Clifford 7 .

En ese contexto histórico, el capitalismo exigía democracia, derechos civiles y políticos, mientras que el socialismo exigía derechos económico-sociales y la no injerencia en los asuntos internos de los Estados, que al final se complementaron para obtener otro nivel de disfrute de los Derechos Humanos. Vemos así, que se configuran de esa manera los derechos económicos y sociales, calificados como derechos de la segunda generación o de "igualdad" que nacen entre los años 60’s y los 70’s del siglo pasado a como lo menciona García ${ }^{8}$, que también reconoce la búsqueda por el bienestar de los pueblos y las personas especialmente por el proceso de la descolonización ${ }^{9}$ de las naciones.

Esos derechos de segunda generación surgieron en esa coyuntura para garantizar la igualdad y la participación de todos los individuos en el ámbito del mercado, consagrando los derechos sociales al trabajo, al salario, al descanso, entre los principales beneficios para los individuos.

Posteriormente, como consecuencia de la evolución de la problemática ambiental, según afirma Jaquenod de Zsögön ${ }^{10}$, es a partir de

7 Clifford, Bob, "New Human Rights Issues: Understanding Their Contentious Rise", Scielo, 2009. Http://Wrerw.Scielo.Org.Co/Scielo.Php?'Script=Sci_Arttex$t$ EPid $=$ S0121-56122009000100002

${ }^{8}$ Garcia, Emilio, Derechos humanos y calidad de vida, Madrid, Universidad Complutense, 1999.

${ }^{9}$ Luego de la firma de la Carta de las Naciones Unidas en 1945, se proclamó en el Derecho Internacional Público, el respeto por el principio de la igualdad de derechos y por la libre determinación de los pueblos, como uno de sus propósitos básicos de la libre determinación de los pueblos (de las antiguas colonias) o territorios dependientes. Luego en las siguientes décadas, específicamente entre los años 60’s y 70’s, más de 80 territorios coloniales se independizaron como resultado de ese principio de la libre determinación. Con ese proceso, mediante el cual esas colonias o territorios ejercieron su derecho a la libre determinación, es conocido como el proceso de descolonización, el cual es reconocido en la Resolución 1541 (XV) de 1961 de la Asamblea General de las Naciones Unidas.

${ }^{10}$ Jaquenod De Zsogon, Silvia, Iniciación al derecho ambiental, España, Dikinson, 1996. 
los años 70's donde se configura la tercera generación de los Derechos Humanos, con la Declaración de Estocolmo ${ }^{11}$, donde se demanda el derecho al medio ambiente o derechos de la solidaridad, que responden a las nuevas tendencias como la globalización, al avance científico técnico y la masificación de la información; entre otros elementos, basados en los valores emergentes de una sociedad globalizada comercialmente que han marcado las últimas décadas del siglo XX, como parte del crecimiento económico de las naciones.

$\mathrm{Al}$ interpretar el fundamento de esos derechos, encontramos que la primera generación radica en la libertad del individuo y el de la segunda en la igualdad, mientras que la tercera se fundamenta en la solidaridad, estrechamente vinculada al propio titular (sujeto del derecho).

Es decir, que la solidaridad es el fundamento que sustenta el carácter expansivo del bien jurídico protegido (medio ambiente), pues no se trata de la libertad de los individuos, sino de un objeto de carácter global que se proyecta en el tiempo (actual, así como del futuro) a nivel intergeneracional y que necesariamente requiere esfuerzos de la cooperación entre los Estados a escala planetaria.

Se aprecia, una dimensión colectiva como el medio ambiente que incluye entre otros, los bienes jurídicos públicos como la atmósfera y los océanos, los cuales, por su carácter, en la medida que el sujeto del derecho lo disfruta ${ }^{12}$, exige a la vez, la responsabilidad jurídica de abstenerse a dañar al sujeto y la de obligación de proteger

${ }^{11}$ Con la Declaración de Estocolmo sobre el Medio Humano y sus Principios de 1972, se conformó el primer cuerpo del conocido Soft Law (legislación suave o blanda), para temas internacionales relativos al medio ambiente. Esta Declaración cuenta con 26 Principios y un plan de acción, los cuales están prescritos en la Resolución de la Asamblea General de la ONU en Doc. A/CONF.48/14/Rev. 1 del 16 de junio de 1972 .

${ }^{12}$ De ello, nace una relación entre el individuo que apela y otro que satisface el requerimiento, es decir alguien exige un medio ambiente saludable y otro responde a la exigencia (Estado), alguien tiene un derecho y otro, tiene la obligación de satisfacer ese derecho exigido. 
el objeto (el sistema climático) del derecho. Se trata entonces de los derechos basados en que toda persona tiene que nacer y vivir en un ambiente sano, no contaminado, por el cual debe perdurar para el relevo intergeneracional de la humanidad.

De tal suerte, que esta generación se inspira, además, en la fraternidad para promover los derechos "solidarios" que faciliten la cooperación, la autodeterminación de los pueblos, la paz mundial, al libre acceso de la información y al medio ambiente sano, íntimamente vinculado a la calidad de vida de las personas ${ }^{13}$.

Asimismo, paulatinamente se han establecido los derechos del buen vivir para promover y garantizar el aprovechamiento responsable y planificado de los recursos naturales; además de integrar diversas facetas humanas necesarias para materializar la idea de la dignidad humana, entre esos: el derecho a la alimentación, al agua, a la comunicación, el respeto a la identidad cultural, la educación, el hábitat adecuado y la vivienda segura, la salud, el trabajo, la seguridad social, entre otros.

Así, por su cercanía a los tiempos actuales son llamados de tercera generación por la naturaleza de las conquistas logradas, debido a que subrayan la responsabilidad personal y social respecto a los bienes jurídicos ambientales que son limitados y finitos a como señaló Pagiola, Bishop y Landell-Mills ${ }^{14}$.

${ }^{13}$ Boyle, Allan, "Human rights or environmental rights? a reassessment", Fordham Environmental Law Review, Vol. XVIII, 2007. Http://Wwrw.Law.Ed.Ac.Uk/Includes/Remote_People_Profile/Remote_Staff_Profile?Sq_Content_Src $=\% 2$ bdxjspwh0dhalm0elmkylmkz3d3cylmxhdy5lzc5hyy 51 ayuyrmzpbgvfzg93 bmxvywqlmkzwdwjsawnhdglvbnmlmkywxzeymiffahotyw5yawdodhnvcmoudmlyb25tzw50ywxyawdodhnhcmvhc3nlcy5wzgymywxspte\%3d; Cecchetto, Sergio, op. cit.

${ }^{14}$ Pagiola, Stefano, Bishop, Joshua y Landell-Mills, Natasha, Selling Forest Environmental Services, Market-Based Mechanisms For Conservation And Development, Londres, Earthscan Publications, 2002, p.96. 
iII. El sistema climático y su vínculo con el Derecho HuMANO DE UN MEDIO AMBIENTE SANO

De ello también nace acá, la justificación moral que razona y se reconoce como una petición justa, debido a que el conjunto de las naciones en la actualidad ha reconocido el principio del desarrollo sostenible en varios instrumentos internacionales, donde se ha respaldado la obligación del Estado en promover, proporcionar y facilitar un medio ambiente saludable.

Tal condición, de satisfacer las necesidades de las futuras generaciones considera la posibilidad cierta y razonable de desarrollar con éxito una vida humana digna, que las generaciones actuales deben, como obligación responsable, legar un sistema climático en equilibrio como herencia oportuna.

Es decir, que el hombre debe de solidarizarse con una responsabilidad intergeneracional para las futuras generaciones y con el medio ambiente por los servicios ambientales ${ }^{15}$ que presta la naturaleza según indica Wunder ${ }^{16}$, siendo esta una obligación ética en un contexto globalizante y de constante avance científico técnico.

Es así que, este vínculo se establece con la conducta de la actual generación junto a los escenarios futuros de la próxima generación, la cual subraya la importancia moral frente a las consecuencias dañinas e irreversibles al dañar los recursos naturales, los bienes jurídicos de carácter público y el medio ambiente, cuya posición es coincidente entre Cecchetto y Boyle.

${ }^{15}$ Los Servicios Ambientales son determinadas prestaciones o beneficios que brindan los bienes jurídicos ambientales que repercuten en beneficio del ser humano y que pueden ser objeto de derechos, principalmente la protección de cuencas hidrográficas, el secuestro y almacenamiento de carbono, la conservación y protección de la diversidad biológica, así como la belleza escénica entre otros, porque tienen un valor comercial significativo, según lo define Pagiola. Pagiola Stefano, et al., op. cit., p. 320.

${ }^{16}$ Wunder, Sven, Pagos por servicios ambientales: principios básicos esenciales, Indonesia, Cifor, 2006, p. 8. 
No obstante, también es conveniente señalar que, la tercera generación de los Derechos Humanos interpela la necesidad de establecer una protección (como un deber de hacer) en el uso sostenible de los recursos naturales y del medio ambiente responsable, a como lo señala Juste Ruiz ${ }^{17}$. Así al considerar lo anterior, vemos entonces que, si los derechos de la tercera generación incluyen al medio ambiente, el sistema climático también está incluido en éste, pues hablamos de un conjunto de elementos sociales, atmosféricos, productivos, económicos, culturales, bióticos y no bióticos que interactúan entre sí, con el ser humano en un espacio y tiempo determinado para la realización de todos los derechos del individuo, en su medio y contexto.

Al revisar los derechos de tercera generación, nace el planteamiento moral, ético y de justicia, de la protección del sistema climático global para legar un desarrollo sostenible del relevo intergeneracional, tal a como lo hemos podido explicar. La reflexión que se presenta al respecto nos indica, que existe el deber moral que nos obliga, partiendo desde el primer momento, que son seres humanos las futuras generaciones y que, por otro lado, no es justo disfrutar y malgastar las condiciones ambientales actuales para únicamente satisfacer nuestras necesidades actuales, en detrimento de las venideras ${ }^{18}$. Si como seres humanos podemos establecer nuestro compromiso, entonces somos capaces de autoimponernos la obligación ética y moral con las futuras generaciones.

${ }^{17}$ Juste Ruiz, José, "El desarrollo sostenible y los derechos humanos", en Curtis, Cristian, Hauser, Denise y Rodriguez Huerta, Gabriela (Coords), Revista De Derechos Humanos. México, Porrúa-Itam, 2005. Https://Revistas.Juridicas.Unam.Mx/Index.Php/ Derecho-Internacional/Article/View/174/285.

${ }^{18} \mathrm{Al}$ respecto, en la Declaración de Río de Janeiro, en la Cumbre de la Tierra de 1992, se estableció que el objeto principal del desarrollo sostenible radica en el bienestar de los seres humanos, a contar con una vida productiva y saludable en armonía con los recursos naturales y el medio ambiente. 
Es ahí donde se sustenta la actuación del Derecho Ambiental, como rama especializada del Derecho, para regular las conductas humanas como ser social, frente al medio que rodea, por su actuación antropogénica y así lograr una interacción armónica y sustentable entre el hombre y el medio ambiente que le rodea, a efecto de que las complejas manifestaciones sociales, económicas, productivas y culturales, mantenga inalterable los procesos naturales o que impacte lo menos posible sobre los recursos naturales y el medio ambiente, según Loperena Rota ${ }^{19}$.

Por ello, he ahí un vínculo con la actual crisis del sistema climático, pues las pasadas y la actual generación, han hecho uso intensivo de los combustibles fósiles, cambio de uso del suelo y la deforestación acelerada, siendo éstas, entre las principales causas del calentamiento global por las consecuencias que este acarrea en el incremento de las emisiones de gases de efecto invernadero, pero poniendo en riesgo a la vez, al propio ser humano.

Deviene así la ignorancia por los daños o que los perjuicios contra el entorno climático y por ende a los recursos naturales, lo cual significa una violación a ese derecho a la vida, que obliga la actuación estatal en implementar acciones públicas de protección, conservación y del uso sostenible de los bienes jurídicos ambientales, para facilitar la adaptación de los ecosistemas, incluyendo la vida de los ciudadanos. Sin embargo, resulta ser que desde la perspectiva antropocéntrica de los Derechos Humanos, ese derecho a un medio ambiente sano supone garantizar que las condiciones del entorno natural y del sistema climático, no se deterioren al punto que ponga en riesgo la vida del ser humano.

Obviamente legar un sistema climático saludable, es al final parte de nuestra obligación como una expresión de prudencia racional frente a la vida en general. Sin embargo, resulta imposible que la

${ }^{19}$ Loperena Rota, Demetrio, "La posición jurídica del ciudadano ante el medio ambiente", Revista Naturzale, No. 12, 2007, p. 59. Http://Wwrw.Euskomedia.Org/Pdfanlt/ Naturzale/12/12057073.Pdf 
generación actual sacrifique su bienestar, porque una actuación responsable considera de manera inevitable incluir a las futuras generaciones, donde concurre acá, el sentido de la responsabilidad con el desarrollo y el progreso; pero también la reciprocidad entre los recursos naturales y su propia regeneración, debido a que es evidente la preservación actual para resolver las demandas a futuro, según el Programa de las Naciones Unidas para el Desarrollo ${ }^{20}$.

De esa manera, el equilibrio ecológico y el disfrute del sistema climático es una proyección al pleno gozo de la realización del ser humano, como una prolongación extensiva del derecho a la vida, con un entorno que favorece la satisfacción de las necesidades de las personas, de la sociedad en general, donde dicho derecho no se reduce expresamente a la simple existencia humana, sino que deberá verse integrado con el goce efectivo de todos los derechos que hacen posible su desarrollo.

Encontramos entonces que, una alteración al sistema climático supone de hecho, una grave lesión al derecho de las personas a vivir y gozar del mismo, manifestándose ese vínculo indisoluble entre sistema climático, crecimiento económico, desarrollo y medio ambiente. Uno no va sin lo otro, como condición inseparable, pues resulta incuestionable que la vida humana necesita contar con un medio ambiente sano que facilite su desarrollo, es decir que el derecho a la vida se disfruta teniendo un derecho ambiental saludable, sustentado en su propia existencia y en la salud humana con la calidad de vida requerida, para el crecimiento de la riqueza en las naciones.

De esa forma, el derecho humano a vivir y a disfrutar de un sistema climático en equilibrio encuentra su razón de ser como un derecho personal, colectivo y a la vez universal, con características que pueden emanar de los derechos civiles y políticos, como

${ }^{20}$ PNUD, Informe sobre desarrollo humano 2014 - resumen -; sostener el progreso humano: reducir vulnerabilidades y construir resiliencia, New York, 2014, p. 12. Http://Wwrw.Undp.Org/ Content/Undp/Es/Home/Librarypage/Hdr/2014-Human-Development-Report/. 
también con los derechos económicos, sociales y culturales. Así al analizar la teoría del calentamiento global y los impactos sobre la población en el mundo actual, se observan que por el incremento de la temperatura se vulneran los Derechos Humanos de la población en las naciones, especialmente en los países subdesarrollados por su poca o escasa capacidad económica para enfrentar los impactos negativos, para este caso nuestra región centroamericana, especialmente Nicaragua.

IV. LA SITUACIÓN NICARAGÜENSE FRENTE AL CAMBIO CLIMÁTICO

En ese sentido, analizamos la perspectiva de la República de Nicaragua frente a esos impactos del cambio climático, la cual se ubica en el centro del istmo centroamericano, siendo una pequeña unidad geográfica de aproximadamente unos $130,375.87 \mathrm{~km}^{2}$, dividido en quince (15) departamentos con ciento cincuenta y tres (153) municipios y dos (2) regiones autónomas.

Con una población aproximada, según el Instituto Nicaragüense de Información para el Desarrollo ${ }^{21}$, de 6,071,048 habitantes, el país mantiene su condición de suministro de materias primas sin ningún valor agregado en la mayoría de las ocasiones, con un alto nivel de dependencia de los préstamos internacionales, deuda externa, importación de productos manufacturados, ingresos muy bajos, condiciones agrícolas benevolentes pero dependientes de la estacionalidad climática, con desempeño bastante interesante en su producción de los principales cultivos como azúcar, café, pesca, minas y otros ${ }^{22}$; que ha permitido la recuperación económica luego del conflicto civil de los años $80^{\prime}$ s.

${ }^{21}$ INIDE, Encuesta De Demografia Y Salud - 2011 Y 2006/2007, Managua, Nicaragua, 2011. Http://Inide.Gob.Ni/Bibliovirtual/Publicaciones.Htm.

${ }^{22}$ Según el BCN (2014) el crecimiento del índice mensual de actividades económicas es de 4,9\% con relación a los años anteriores. 
No obstante, el Producto Interno Bruto (PIB), según el Banco Central de Nicaragua (BCN, 2013) fue de 11,255.6 millones de dólares, con una inflación controlada que varía entre el 6.6\% (2012) y un 5.7\% (2013), con un ingreso nacional bruto per cápita en 2013 de 1,780.3 dólares/anuales, aparejado con el PIB per cápita nacional.

De esa manera, el país es un productor agrícola neto (incluyendo la ganadería, silvicultura, pesca y la minería), con un volumen total de aproximadamente unos U \$ 3,340 millones de dólares en el $2013^{23}$, con capacidad productiva para satisfacer la demanda nacional y la exportación, limitado por no dar un valor agregado en las materias primas que produce, lo que estrecha su participación en el comercio internacional.

A pesar de lo mencionado, el flagelo de la pobreza, la desigualdad y el crecimiento económico ${ }^{24}$ continúan siendo retos insoslayables para la sostenibilidad y el desarrollo humano sostenible. Empero a lo anterior, a pesar de las dificultades, Nicaragua avanza lentamente en el concierto internacional, superando unas décadas de crisis bancarias, productivas, desastres naturales, guerra civil y otros, impulsando el crecimiento de sus exportaciones, haciendo uso de la naturaleza como un medio de subsistencia, pero cargando con una población que crece paralelamente con deuda (pública y externa), que al final ejerce mayor presión sobre los recursos naturales, vulnerando el beneficio al derecho de un medio ambiente sano.

${ }^{23}$ BCN, Índice mensual de actividad económica, managua, Nicaragua, 2014 Http:// Wwre.Bcn.Gob.Ni/Publicaciones/Periodicidad/Mensual/Imae/0914/Informe.Pdf.

${ }^{24}$ Las instituciones financieras internacionales como el Banco Interamericano de Desarrollo (BID, Indicadores De Riesgo De Desastre Y De Gestión De Riesgos: Programa Para América Latina Y El Caribe: Nicaragua, Washington, USA, 2015. Https://Publications.Iadb. Org/Handle/11319/6973?Locale-Attribute=Es) y el Banco Mundial (BM, Nicaragua: informe sobre la pobreza 1993-2005, Washington, USA, 2008 Https://Bjcu.Wordpress.Com/ Tag/Banco-Mundial/), coinciden que casi un total del $60 \%$ de la población total, se encuentra en situación de pobreza (bajo la metodología de línea de los ingresos), aunque se ha logrado la reducción de la pobreza extrema en un par de dígitos, con niveles altos en la tasa de mortalidad infantil y materna, con una esperanza de vida de 74 años. 
Aunque el país posee exuberante riqueza en su patrimonio natural, suelos fértiles, recursos hídricos, diversidad biológica, volcanes, recursos marino-costeros, potencial energético renovable disponible, población relativamente joven y condiciones productivas interesantes para empujar el crecimiento económico, se enfrenta a la vulnerabilidad y a las amenazas por desastres naturales e inducidos por el calentamiento global ${ }^{25}$, agudizando la estructura socioeconómica y productiva en la extracción de los recursos naturales y la depredación del medio ambiente en general.

V. LA VULNERABILIDAD, LA GOBERNANZA AMBIENTAL Y LA GESTIÓN CLIMÁTICA NICARAGÜENSE

Así pues, en Nicaragua se nos presenta como resultado un modelo de desarrollo económico con sistemas productivos insostenibles, que provocan aumento gradual de emisiones de gases de efecto invernadero ${ }^{26}$, incremento de la contaminación de las fuentes hídricas principalmente, del cual se acrecienta la vulnerabilidad nacional, cuando observamos la situación productiva y socio-económica. Una muestra de la vulnerabilidad por señalar, se agudiza con la pérdida del recurso forestal, del cual Nicaragua presenta entre 1950 al 2000 una pérdida forestal aproximada de 29,628.33 $\mathrm{km}^{2}$ de bos-

${ }^{25}$ Acudimos concluir entonces que, Nicaragua posee una vocación agropecuaria, forestal y pesquera, pero que dichos sectores dependen estrechamente de los impactos del incremento de la temperatura global, en particular las alteraciones en los recursos hídricos y de la precipitación, en el desarrollo nicaragüense según el Banco Mundial. $\mathrm{BM}$, op cit.

${ }^{26}$ Conocidos por su influencia en el calentamiento global, los gases de efecto invernadero resultan imprescindibles para mantener la temperatura del planeta, pero la actividad humana ha aumentado su composición en la atmósfera y ha alterado su equilibrio natural. Estos han sido reconocidos por la Convención Marco de las Naciones Unidas sobre Cambio Climático (CMNUCG), como la fuente del calentamiento global, porque la concentración incrementada de estos gases en la atmósfera está alterando el equilibrio natural del clima. 
ques, equivalentes a 59,267 hectáreas anuales según el Ministerio del Ambiente y Recursos Naturales ${ }^{27}$. A ello se debe agregar que, entre el período del 2000 al 2010, la tasa anual de pérdida forestal se estima en 70,000 hectáreas anuales según el BID ${ }^{28}$ con la cual se contribuye a la vulnerabilidad actual climática nacional.

\section{Un PAís CON MÚLTIPLES AMENAZAS}

El país presenta altos niveles de multiamenazas entre las regiones geográficas que agudizan la situación de vulnerabilidad social, económica y ambiental, siendo entre las principales amenazas que podemos señalar: las geológicas, sísmicas, naturales e inducidas por la actividad antropogénica según las diversas fuentes consultadas, que complementan el alto nivel de vulnerabilidad que tiene la nación más grande en superficie de Centroamérica.

No obstante, de acuerdo a fuentes especializadas también demuestran que Nicaragua debe ser considerado como país altamente vulnerable frente al cambio climático ${ }^{29}\left(\right.$ Germanwatch $\left.^{30}\right)$ a los de-

${ }^{27}$ MARENA, Informe del estado ambiental en Nicaragua, Managua, 2001. Http://Wwre. Pnuma.Org/Deat1/Pdf/Geonicaragua2001.Pdf.

${ }^{28} \mathrm{BID}$, Vulnerabilidad y adaptación al cambio climático: diagnóstico inicial, avances, vacíos y potenciales líneas de acción en Mesoamérica, Washington. USA, 2010, p. 49. Https://Publications.Iadb.Org/Handle/11319/1761?Locale-Attribute $=$ En.

${ }^{29}$ BID, op. cit; Banco Interamericano de Desarrollo / Banco Mundial, Perfil del riesgo de desastres de Nicaragua, Washington, USA, 2010. Https://Publications.Iadb.Org/Bitstream/Handle/11319/6973/Indicadores_Riesgo_Desastre_Gestion_Riesgos_Programa_America_Latina_Caribe_Nicaragua.Pdf; Germanwatch, "Who Suffers Most from Extreme Weather Events? Weather-Related Loss Events In 2012 And 1993 To 2012", Global Climate Risk Index 2014, 2013. Http://Germanwatch.Org/De/Download/8551.Pdf.; Germanwatch "Who Suffers Most from Extreme Weather Events? Weather-Related Loss Events In 2013 And 1994 To 2013”, Global Climate Risk Index 2014, 2014. Http://Germanwatch.Org/En/Cri; y MARENA, Segunda comunicación nacional ante la convención marco de las Naciones Unidas sobre cambio climático, Managua, 2008. Http://Unfccc.Int/National_Reports/Non-Annex_I_Natcom/Submitted_Natcom/Items/653.Php.

${ }^{30}$ Organización No Gubernamental (ONG) que ha desarrollado el Índice de Ries- 
sastres naturales, así como a los eventos extremos originados por el calentamiento global, el cual complementa negativamente la vulnerabilidad de la población nicaragüense.

\section{De la puntuación del riesgo.}

Vemos que, a través del Índice de Riesgo Global Climático en las décadas de 1992 al 2011, Nicaragua es considerado con el mayor índice negativo asociado a los eventos extremos, como una consecuencia del incremento de la temperatura global. De ahí que, el país se ubica entre los primeros diez países de América Latina y el Caribe, afectados por los impactos de los eventos hidrometeorológicos, climáticos y geológicos según Germanwatch ${ }^{31}$, debido a la exposición a múltiples amenazas y las vulnerabilidades que se han incrementado en las últimas décadas.

Así Nicaragua luce como un país altamente afectado por el cambio climático en los últimos decenios, al ser evaluada por instituciones extranjeras, a pesar que la responsabilidad histórica en las emisiones del paradigma es y ha sido un gravamen que deberían de asumir los países industrializados, por la cual se niega el disfrute del Derecho Humano a un sistema climático sostenible. Empero a lo anterior, la huella de carbono de Nicaragua es tan baja de apenas el 0,03\% según el MARENA ${ }^{32}$, pero ha sido afectado y se verá afectado con consecuencias funestas para el crecimiento y el desarrollo

go Climático Global (IRC), el cual relaciona el nivel de exposición y el grado de vulnerabilidad que poseen los países frente a los fenómenos climáticos extremos, que debe entenderse como una realidad advertida para prepararse ante dichos eventos con más periodicidad, mayor frecuencia y de un incremento en la magnitud en el futuro próximo. Sin embargo, hay otros factores como como terremotos, erupciones volcánicas (de origen geológico) o tsunamis, cuyos datos también están disponibles, pero no son utilizados para fines de la configuración del IRC en este contexto, en vista que estos no dependen ni están relacionados con el cambio climático.

${ }^{31}$ Germanwatch... 2013, op cit.

${ }^{32}$ MARENA, op. cit., p. 5. 
económico por los impactos del cambio climático, para afirmar que la situación de vulnerabilidad se agravará con una población con casi la mitad $(47,9 \%)$ de la población sometida en el círculo de la pobreza ${ }^{33}$.

También el BID $^{34}$ que ha diseñado el Índice de Vulnerabilidad Prevalente (IVP por sus siglas), certifica que Nicaragua presenta la mayor vulnerabilidad prevalente en la región centroamericana, reafirmando que el Estado nicaragüense no estaría en capacidad financiera para cubrir los pasivos fiscales asociados al impacto de un evento hidrometeorológico extremo de magnitud conforme al Índice de Déficit por Desastres (IDD) del estudio realizado por el BID ${ }^{35}$.

A pesar de lo anterior, según el PNUD $^{36}$ en el Índice de Desarrollo Humano, Nicaragua ha logrado dar algunos saltos cualitativos en términos de salud, educación e ingresos que superan en alguna medida la vulnerabilidad socioeconómica y de los Derechos Humanos de su población. Pero, la vulnerabilidad en todos los sentidos, ha aumentado como consecuencia de la migración, el cambio de uso del suelo, el deterioro ambiental, la deforestación, el crecimiento urbano, la falta de un ordenamiento territorial, los asentamientos humanos y otros, que perjudican de manera acentuada la situación en este sentido.

${ }^{33}$ En relación al objeto del presente, encontramos que la pobreza es generalmente considerada como una de las causas más importantes de la vulnerabilidad frente a las amenazas a los desastres, con fundamento en el hecho de que los habitantes en condición de pobreza, suelen tener una capacidad muy limitada (de recursos) para enfrentar los riesgos a los eventos climáticos y naturales, lo que por consiguiente, soportan una carga desproporcionada de los impactos producto de desastres, conflictos, sequías, desertificación, deslizamientos, entre otros asociados a los efectos negativos del calentamiento global. BID... 2010, op. cit.; INIDE, op. cit.

${ }^{34}$ BID... 2010, op. cit., p. 54.

${ }^{35}$ BID... 2015, op. cit., p. 53.

${ }^{36}$ PNUD, op. cit., p. 24. 
3. De la gobernanza ambiental y la gestión climática.

El futuro a corto plazo y de forma inmediata, exige medidas que contrarresten la vulnerabilidad frente al impacto de los eventos extremos y se favorezca la adaptación mejorando la resiliencia climática local, es decir la gobernanza ambiental para el disfrute de un sistema climático equilibrado y por ende de los Derechos Humanos.

De ahí que al ser un país subdesarrollo, Nicaragua delinea su gobernanza ambiental y de gestión climática, así como del riesgo ante los desastres naturales inducidos por el mismo, con varias políticas y estrategias. De ello, sin embargo, sobresale el Plan Nacional de Desarrollo Humano (PNDH), el cual define lineamientos generales de políticas para el desarrollo, el combate a la pobreza y el crecimiento económico.

Este instrumento de política pública (el PNDH), nació en 2008 y fue presentado para el quinquenio entre 2008-2012, siendo luego actualizado para el 2012-2016, lo que se constituye en sí mismo, como el principal instrumento de orientación estratégica institucional para el desarrollo, estableciendo entre los primordiales lineamientos relativos al ámbito que nos ocupa: i) protección al medio ambiente; ii) de la participación en las negociaciones ambientales y de cambio climático; iii) en la Costa Caribe; la defensa y protección del medio ambiente, así como de adaptación al cambio climático y de gestión del riesgo; iv) la seguridad y la soberanía alimentaria en un contexto climático cambiante y v) la protección de la Madre Tierra, adaptación y gestión integral del riesgo ante los desastres naturales.

El PNDH a la fecha, es el instrumento de política institucional que incorpora varios ejes fundamentales, pero específicamente cuenta con el de mitigación, adaptación y gestión de riesgo ante el cambio climático. Así al comparar el primer instrumento generado con el segundo PNDH (2012-2016), encontramos un salto cualitativo con indicadores, metas de seguimiento y evaluación e instrumen- 
tos para la medición del desempeño que, a pesar de ser instrumento público muy alegórico de lo que se realizará, motiva la ejecución de acciones en este ámbito.

Además, el Gobierno de Nicaragua también cuenta con una Estrategia Nacional Ambiental y de Cambio Climático (ENACG), como lineamiento de política pública específicamente a éste ámbito, que aborda el problema climático desde una perspectiva holística y global, la cual propone cinco lineamientos estratégicos: i) la educación ambiental para la vida; ii) la defensa y protección de los recursos naturales; iii) la conservación, recuperación, captación y cosecha de las fuentes de agua; iv) la mitigación, adaptación y gestión del riesgo ante el cambio climático y v) el manejo sostenible de la tierra.

Aunque se reconoce que, la estrategia mencionada no establece acciones directamente al tema de mitigación y adaptación, toca el tema de manera general del clima cambiante de forma transversal, cuenta con orientaciones de acción, dentro de estrategias globales (no locales) sin priorizar ni la evaluación exhaustiva de los sectores de la vida socio productiva y económica de la nación, lo que se considera como limitantes.

Es decir, al no contar con una evaluación constante, el instrumento se ve limitado, pues no ofrece la hoja de ruta a seguir desde el punto de vista local, para facilitar las opciones y medidas de adaptación en los municipios de la nación. Así visto, la ENACG no presenta costos ni define una priorización de sectores, pues carece de mecanismos de coordinación interinstitucional para manejar las interrelaciones sectoriales del manejo de los bienes jurídicos ambientales y de los procesos en el país, con una autoridad competente que asuma el liderazgo, resultando de alguna manera, una alegoría al deseo de implementar acciones, lo que exige una obligación imperante para integrarse en el presupuesto nacional.

Como instrumento la ENACG es un paso sustantivo, con limitaciones, definida así por el gobierno a través de los proyectos de 
inversión pública para el desarrollo productivo y económico. No obstante, se reconoce entonces que, el tema de los impactos y las consecuencias que trae el cambio climático (largo plazo) para facilitar la adaptación, van más allá de uno o más proyectos puntuales, es decir; esto debe ser una política de Estado que trasciende un plazo gubernamental, donde los proyectos son instrumentos para lograr algo en un espacio temporal, mientras otros elementos complementan la estrategia gubernamental de éxito en una política nacional y local.

Sin embargo, tanto la estrategia como el instrumento de política pública y la implementación en muchas ocasiones, no se logran articular al nivel territorial, pues la problemática, en la mayoría de todos los ámbitos, sobrepasa la capacidad de la autoridad local en el territorio. Es decir, que la problemática ambiental genera, directa o indirectamente, múltiples efectos e impactos de naturaleza física, ecológica, económica, cultural y social en el territorio; donde nos encontramos con los municipios más vulnerables, los cuales están ubicados en los niveles de pobreza en el país.

vi. Hacia una propuesta normativa climática para ENFRENTAR LA PROBLEMÁTICA

A pesar que, el conjunto de instrumentos de política, estrategias, planes, leyes y proyectos en implementación (o por ejecutar), dependen de esa articulación entre el nivel central y el nivel territorial, para enfrentar tanto el riesgo a los desastres inducidos por los efectos del cambio climático, así como la amenaza al mismo para su implementación, van vulnerando paulatinamente el derecho a un sistema climático global sano para las futuras generaciones.

Así que lograr una gestión exitosa para enfrentar los riesgos a los desastres y mitigar la vulnerabilidad a los efectos negativos del calentamiento global, implica poner en práctica una serie múltiple de políticas públicas y decisiones, con un contenido de justicia social 
y ambiental, coordinados a nivel institucional, ajustados a la realidad propia de los diversos municipios, porque la capacidad para implementar las leyes, políticas y normativas es un reto pendiente para una buena gobernanza en el país en todos los sentidos, para un pleno gozo de los Derechos Humanos.

Vemos entonces así que, la carta fundamental de la República de Nicaragua, es decir la Constitución (Cn) nicaragüense, acoge el principio de jerarquía normativa, estableciendo disposiciones constitucionales aplicables a la problemática que nos toca, específicamente con: el acceso a un ambiente sano, como un derecho humano fundamental de todos los nicaragüenses; la obligación del Estado nicaragüense, en preservar, conservar, desarrollar, manejar y rescatar el ambiente y los recursos naturales, así como explotarlos racionalmente; la consideración de patrimonio nacional, a todos los recursos naturales que se encuentran en el territorio, el cual debe incluir el clima como parte de estos; la atribución del Estado para celebrar contratos de explotación racional de dichos recursos, cuando el interés nacional así lo requiera; el deber del Estado de solicitar y tomar en cuenta la opinión de los gobiernos municipales, antes de autorizar un contrato o concesión de explotación racional de los recursos naturales; la obligación de contar con la aprobación del Consejo Regional Autónomo correspondiente, de las comunidades de la Costa Caribe, en caso de otorgar una concesión o contrato de explotación de los recursos naturales en dicha jurisdicción geográfica; la obligación social del derecho de informar y su ejercicio, con estricto respeto a los principios constitucionales, el cual no debe ser sujeto a censura, sino a responsabilidades posteriores.

Encontramos pues que, las políticas ambientales de Nicaragua se sustentan especialmente con el artículo $60(\mathrm{Cn})$ y en los principios del PNDH 2012-2016, el cual pretende restituir el principio fundamental de los Derechos Humanos, que todo individuo debe gozar de un ambiente sano. 
De igual forma, dicho artículo establece la obligación estatal de la preservación, conservación y rescate del medio ambiente y de los recursos naturales, viendo en este artículo, el derecho inherente de la presencia de la persona, es decir el derecho a la vida, siendo el punto de partida al derecho a vivir en condiciones que hagan viable y posible la existencia humana.

Luego se fundamenta también con la cuantificación del patrimonio nacional, de lo cual los recursos naturales son parte del mismo, a como rola en el artículo $102(\mathrm{Cn})$, responsabilizando al Estado nicaragüense por la preservación del medio ambiente, la conservación, desarrollo y explotación racional de los recursos naturales. Dicha responsabilidad se manifiesta, en asegurar el desarrollo humano sostenible con la propia dinámica del medio ambiente y los recursos naturales de forma equilibrada.

Adicionalmente según el artículo 180 (Cn), le corresponde al Estado garantizar a las comunidades de la Costa Caribe, el disfrute de sus recursos naturales, la efectividad de sus formas de propiedad comunal y la libre elección de sus autoridades en las comunidades, según sus formas de organización social, costumbres y tradiciones históricas.

Observamos entonces, que la estructura normativa en la gestión climática nace desde la carta magna, aunque no referida especialmente así, es menester expreso con el tema de la conservación, protección, uso y manejo de los recursos naturales y del medio ambiente para el desarrollo nacional.

También como parte de la normativa institucional vigente, se debe mencionar la aprobación de los instrumentos internacionales tales como: la Convención Marco de las Naciones Unidas sobre el Cambio Climático ${ }^{37}$ y del Protocolo de Kyoto $^{38}$, siendo estos los

${ }^{37}$ Ratificado mediante Decreto no. 50-95, publicado en La Gaceta no. 199 del 24 de octubre de 1995.

${ }^{38}$ Ratificado mediante Decreto no. 94-99, publicado en La Gaceta no. 168 del 2 de septiembre de 1999 . 
principales, aunque a nivel de la integración regional en el Sistema de Integración de Centroamérica (SICA), también existen normativas regionales comunitarias que se han incorporado al marco legal vigente de esta materia como el Convenio Regional sobre Cambios Climáticos (1993), la Alianza Regional para el Desarrollo Sostenible (1994), la Estrategia Regional de Cambio Climático (2011) y la Política Centroamericana de Gestión Integral de Riesgo (2011), entre los más importantes.

Vemos así en ese contexto, que a nivel nacional encontramos: la Ley General del Medio Ambiente y los Recursos Naturales (Ley no. 217 y sus reformas) que establece el estamento de la gestión ambiental, así como otras que complementan tales como, la Ley no. 620 (Ley de Aguas Nacionales) y la Ley no. 462 (Ley de Conservación, Fomento y Desarrollo Sostenibles del Sector Forestal) entre las principales, encontrando que la gestión climática no cuenta con un instrumento normativo específico en esta materia de forma exclusiva, pues la temática se ha visto como un tema transversal a considerar.

Sin embargo, en la actualidad el fenómeno climático ha sobrepasado los límites de la actuación del ser humano sobre la composición atmosférica, lo cual obliga a considerarse en el conjunto normativo y su posterior cumplimiento a través de un instrumento legal. El vacío legal de no contar con un instrumento normativo en esta materia, brinda la oportunidad de su creación, con el fin de facilitar tanto la mitigación al cambio climático como la adaptación a los efectos y consecuencias que trae consigo.

A partir de ahí, encontramos que Nicaragua por su propia ubicación geográfica y por las condiciones geológicas, es parte de una región altamente vulnerable, la cual a la vez, es frecuentemente afectada por los fenómenos naturales derivados del cambio climático, que también constituyen una amenaza latente, poniendo en peligro y en riesgo a su población, siendo referido a las erupciones volcánicas, la sismicidad, junto con los fenómenos meteorológicos peligrosos como tormentas tropicales, las sequías y huracanes, entre otros. 
Considerando lo anterior, los instrumentos normativos de la nación, requieren modernizarse, actualizarse o mejorarse en un marco legal regulatorio, que tutele tanto a los bienes jurídicos ambientales como a los ciudadanos en general. Incluso, ante un vacío frente a determinados problemas, la ciencia jurídica a través del Derecho, está obligado a intervenir con las exigencias que plantea dicha problemática para la sociedad, utilizando de otras fuentes para satisfacer determinada situación jurídica.

He ahí nuestra posición, partiendo del hecho que Nicaragua es un país con un alto nivel de vulnerabilidad, multiamenazas y de riesgo, que ha sido y será impactado por el cambio climático con mayor rudeza, que posee cierta dispersión legal y normativa en determinados instrumentos de política pública y por el objeto, de incrementar su capacidad adaptativa para el manejo de la problemática del sistema climático, se considera oportuno sugerir una propuesta que fortalezca su posición en cuanto a los impactos negativos por un lado y que facilite la resiliencia climática frente a los escenarios que el país enfrentará hacia un futuro próximo.

Comenzaríamos por enunciar que, el objeto de una propuesta de ley para mitigar la vulnerabilidad, facilitar la adaptación ante los efectos del cambio climático y de mitigación a los gases de efecto invernadero, sería conocida como ley de cambio climático, la que debería estar sujeta al marco jurídico en vigencia, de orden público e interés general; cuyas disposiciones son de observancia obligatoria en todo el territorio en establecer las regulaciones necesarias de política pública para prevenir, planificar y responder de manera urgente, adecuada, coordinada y sostenida a los impactos del cambio climático en el país, con la finalidad de coadyuvar a que el República de Nicaragua a través del Gobierno central, entidades descentralizadas, entes autónomos, municipalidades, consejos regionales y la sociedad civil organizada; adopten prácticas que reduzcan la vulnerabilidad de la población y los ecosistemas, garantice el derecho a un medio ambiente saludable; promuevan la capacidad adaptativa, 
permitan la mitigación de los efectos del calentamiento global y propicie tanto la resiliencia climática, como el desarrollo humano sostenible.

Como ámbito de aplicación, el modelo propuesto tendría observancia en general para todo el territorio nacional de la República, por consiguiente, sería de carácter obligatorio para todos los ciudadanos nacionales, entidades públicas o privadas, autónomas y descentralizadas.

Habría a continuación que definir los conceptos a contener, entre los cuales deberán describirse como principales: adaptación, calentamiento global, cambio climático, cambio de uso del suelo, capacidad adaptativa, captura de carbono, comunicación nacional, convención, corredores biológicos, degradación, deforestación, efectos adversos del cambio climático, emisión, efecto invernadero, escenarios, fomento de las capacidades, fuentes emisoras, gases de efecto invernadero, inventarios nacionales, línea de base, mecanismo de desarrollo limpio, mitigación, ordenamiento territorial, protocolo, reforestación, reservas silvestres privadas, resiliencia, resiliencia climática, riesgo climático, servicios ambientales, sistemas de restauración ambiental, sumideros, variabilidad climática, variabilidad extrema, vulnerabilidad, entre otros; para esclarecer y definir efectivamente cómo debería entenderse cada concepto previsto en la propuesta de ley de cambio climático.

Luego, dicho instrumento definiría como objetivos generales: a) fomentar la cultura preventiva que permita mitigar en lo posible, las consecuencias del cambio climático; b) establecer las acciones pertinentes que converjan con las medidas de adaptación y mitigación para enfrentar los impactos de los eventos extremos hidrometeorológicos; c) contribuir con las bases de coordinación interinstitucional entre las dependencias del Gobierno, las municipalidades y consejos regionales en materia de riesgo, vulnerabilidad, adaptación y mitigación ante el calentamiento global; d) reducir la vulnerabilidad definiendo una política estatal en materia de adaptación 
y mitigación ante el cambio climático; e) fomentar la educación, la investigación y la transferencia de tecnología e innovación en materia de adaptación y mitigación; f) promover la transición hacia una economía competitiva, sustentable y de bajas emisiones, que promuevan las bases para una concertación entre sector privado, sector público y ciudadanía en general; g) construir las capacidades adaptativas frente a la variabilidad climática y consolidar a mediano plazo las opciones de resiliencia climática y de adaptación en el crecimiento económico junto al desarrollo humano sostenible.

Como principios rectores en un segundo capítulo, dicho instrumento además de los principios contenidos en la Constitución de la República de Nicaragua, de los tratados internacionales ratificados por el Estado nicaragüense en materia de cambio climático y de la Ley no. 217 y sus reformas, debería contener entre otros: precautorio, prevención, quien contamina paga; transparencia; acceso a la información; participación ciudadana; instrumentos económicos para la mitigación; adaptación y reducción de la vulnerabilidad ante el cambio climático; desarrollo sostenible; cooperación internacional e igualdad soberana sobre los recursos naturales.

Se crearía el Instituto Nacional de Biodiversidad, Silvicultura y de Cambio Climático, mediante el capítulo tercero, como una entidad pública descentralizada, adscrita al MARENA, con personería jurídica, patrimonio propio, autonomía administrativa en su gestión operativa, de conformidad al marco legal vigente, que se convertiría en sucesor del INAFOR ${ }^{39}$, el cual podrá abrir oficinas en los departamentos o su representación será realizada a través de las delegaciones territoriales que posee MARENA.

Así de esa manera, dicho instituto tendría por objeto entre las principales funciones, las siguientes que se describen: a) C o o r d i nar y realizar los estudios, programas, talleres de capacitación, líneas de base y proyectos de investigación científica o tecnológica

\footnotetext{
${ }^{39}$ Instituto Nacional Forestal.
} 
con las instituciones académicas (sean estas públicas o privadas, nacionales o extranjeras) en materia de cambio climático, protección al ambiente, a la biodiversidad, el patrimonio forestal y la preservación junto a la restauración del equilibrio ecológico a nivel nacional, territorial o regional; b) Facilitar y brindar el apoyo técnico y científico al MARENA, MAG, ENACAL, INETER, MINSA ${ }^{40} \mathrm{e}$ INAFOR, para formular, conducir y evaluar la política nacional en los ámbitos sectoriales que incluyan transversalmente el equilibrio ecológico, la vulnerabilidad, la adaptación, la mitigación, la gestión de los riesgos y la amenaza al cambio climático, así como la protección del medio ambiente; c) Formular, controlar y evaluar los instrumentos de política pública, sean éstos la política nacional en materia de cambio climático; la estrategia nacional sobre cambio climático; los planes nacionales de adaptación y de mitigación; los inventarios nacionales; los escenarios climáticos; las comunicaciones nacionales ante la CMNUCC; la divulgación de los estudios realizados; los contenidos de educación ambiental en esta materia; los proyectos y la transmisión de experiencia; la creación de capacidades nacionales tanto a nivel nacional, local y regional a través de las universidades; así como de publicar y difundir los resultados con la participación de la sociedad civil organizada; d) Elaborar, actualizar y publicar el atlas nacional de riesgo, vulnerabilidad y amenazas frente al cambio climático a nivel municipal, regional y nacional, creando las capacidades en dichos territorios de manera coordinada con el Sistema Nacional de Prevención, Mitigación y Atención a los Desastres (SINAPRED); e) Establecer los procedimientos para realizar consultas públicas a la sociedad civil, los sectores público y privado, con el fin de formular la Estrategia Nacional y el Plan Nacional de Adaptación y Mitigación al cambio climático; f) Promover y difundir criterios, metodologías y tecnolo-

${ }^{40}$ Ministerio de Agricultura y Ganadería (MAG), a la Empresa Nacional de Aguas, Acueductos y Alcantarillados (ENACAL), el Instituto Nacional de Estudios Territoriales (INETER) y el Ministerio de Salud (MINSA). 
gías para la conservación y el aprovechamiento sustentable de los recursos naturales, mediante los contenidos educativos a través del MINSA, IND y el $\mathrm{MED}^{41}$; g) Coadyuvar en la preparación de recursos humanos calificados a través del Consejo Nacional de Universidades (CNU), a fin de atender la problemática nacional con respecto al medio ambiente y el cambio climático, aprovechando las capacidades locales existentes; h) Proponer, impulsar y apoyar técnicamente la elaboración de normas en materia de ordenamiento ecológico, conservación de ecosistemas y especies de vida silvestre, contaminación y calidad ambiental, de colecta de especímenes con fines científicos y de investigación, de aprovechamiento para su utilización en biotecnología, acceso a recursos genéticos, así como para la utilización confinada, el manejo, la movilización y la liberación experimental, en programas piloto y comercial, de organismos genéticamente modificados, incorporando en los instrumentos de política pública los criterios de mitigación y adaptación al cambio climático; i) Fomentar la investigación científica y tecnológica, el desarrollo, transferencia y despliegue de tecnologías, equipos y procesos para la mitigación y adaptación al cambio climático, promoviendo la difusión de los contenidos a todos los niveles educativos, así como realizar campañas de educación e información para sensibilizar a la población en general sobre los efectos, consecuencias e impactos del cambio climático; j) Establecer, poner en marcha y actualizar constantemente el Sistema de Información sobre el Cambio Climático, acoplado con el Sistema Nacional de Información Ambiental (SINIA/MARENA), así como ponerlo a disposición del público en los términos de la presente Ley y demás disposiciones jurídicas aplicables; donde se difundan las investigaciones, estudios realizados, contenidos de educación ambiental, mapas de riesgo y amenazas, inventarios nacionales, así como toda aquella informa-

${ }^{41}$ Ministerio de Salud (MINSA), Instituto Nacional de Deportes (IND) y Ministerio de Educación (MED) 
ción que se considere pertinente para la sociedad en general; entre los principales.

Así visto, el patrimonio de esa institución se sugiere que estaría integrado por: i) los bienes muebles e inmuebles, así como por los derechos y obligaciones que le transfiera el Gobierno de la República, los municipios o los consejos regionales; ii) las donaciones, legados y aportes que otorguen particulares o cualquier institución pública o privada, nacional o internacional; iii) las adquisiciones, créditos, préstamos y cooperación técnica (no reembolsable) en recursos en efectivo o en especie, que se obtenga de alguna dependencia o entidad pública, institución privada u organismos nacionales o internacionales, de conformidad con las disposiciones jurídicas aplicables; iv) los bienes muebles e inmuebles, las acciones, derechos o productos que adquiera por cualquier título; v) los recursos que provengan del Presupuesto General de la República que le sean asignados; vi) los recursos que se obtengan por la comercialización, depósitos o derechos de obras publicadas, certificaciones u otros que correspondan según la legislación vigente y vii) los bienes y derechos que se establezcan por las leyes y reglamentos que provengan de otros fondos o aportaciones.

Dicho instituto tendría una junta directiva, como máxima autoridad, que estará presidida por el Ministro del MARENA e integrada por el sector público, con los titulares de los Ministerios (MAG, MINSA, INAFOR, MEM, MHCP, INETER ${ }^{42}$ ), el Director Ejecutivo del Instituto (que actuaría de secretario de la junta) y la Secretaría de Políticas Públicas de la Presidencia de la República, donde cada titular deberá nombrar un suplente jerárquico; así como dos representantes del sector privado a propuesta del Presidente de la República; gozando de voz y voto en las sesiones semestrales

${ }^{42}$ Ministerio de Agricultura (MAG), Ministerio de Salud (MINSA), Instituto Nacional Forestal (INAFOR), Ministerio de Energía y Minas (MEM), Ministerio de Hacienda y Crédito Público (MHCP), Instituto Nicaragüense de Estudios Territoriales (INETER). 
y que sus decisiones serán tomadas por mayoría de los presentes participantes.

Además, se integrarían a dos representantes del sector académico universitario, designados por el Presidente de la nación y su reglamento determinaría las unidades administrativas, facultades y funciones para cumplir con el objeto final de la entidad.

Considerando que existe un par de ámbitos separados (adaptación y mitigación) pero que se complementan, el siguiente capítulo será destinado exclusivamente a la temática de la adaptación. A partir de ésta, se deberá establecer una política nacional de adaptación frente al cambio climático con diagnóstico, con instrumentos de planificación, elementos de medición y monitoreo, con mecanismos de validación y verificación de aplicación territorial.

Entre los principales objetivos que dicho capítulo de la adaptación deberá contener, a manera de sugerencia con: a) reducir la vulnerabilidad de los asentamientos humanos en el territorio nacional y de los ecosistemas frente a los efectos negativos del calentamiento global; b) promover la resiliencia climática de los sistemas naturales y humanos; c) mitigar y disminuir los riesgos, daños y consecuencias climáticas a los ecosistemas, tomando en cuenta los escenarios climáticos; d) identificar la vulnerabilidad, riesgos y capacidad adaptativa en la transformación de los sistemas ecológicos, físicos y sociales, aprovechando las oportunidades generadas que se consideran benéficas por nuevas condiciones climáticas en el territorio; e) establecer los mecanismos de atención inmediata y expedita en zonas de riesgo e impactadas por los efectos climáticos adversos; f) facilitar y promover la seguridad alimentaria, la productividad agrícola, ganadera, pesquera, acuícola, la preservación de la biodiversidad, los bosques y de los recursos naturales en general.

Luego para el caso de la mitigación, deberá incluir como objetivos: a) incluir mecanismos y elementos económicos (incentivos) en la planificación y política nacional de cambio climático, con el fin de reducir las emisiones de los diferentes sectores, considerando las comunica- 
ciones nacionales, la línea de base por sector (económico, productivo e industrial) y los tratados internacionales suscritos por Nicaragua en esta materia; b) promover el fortalecimiento de capacidades nacionales, mediante talleres, seminarios, estudios, investigaciones aplicadas para la mitigación de emisiones del sector forestal, facilitando la adaptación; c) conseguir, diseñar y elaborar las propuestas de proyectos, programas y recursos de las fuentes externas disponibles en materia de cambio climático, así como de mecanismos bilaterales con países interesados en la mitigación por el sector forestal, energía, transporte y de residuos; d) establecer el sistema de compensación económica por servicios ambientales de manera voluntaria para los participantes del sector privado o de los municipios u otros, que implementan acciones de mitigación al cambio climático; e) reducir las emisiones por sectores en proyectos nacionales, que fomenten la transición a una economía baja de emisiones, de forma sustentable, competitiva, incluyendo mecanismos de mercado, incentivos, compensaciones y otras alternativas que fortalezcan las medidas específicas de mitigación, promoviendo la competitividad y la transferencia de tecnología; f) promover de manera gradual la sustitución del uso y consumo de los combustibles fósiles por fuentes renovables de energía, en la generación de electricidad a través del uso de fuentes renovables de energía; las prácticas de eficiencia energética, el desarrollo de transferencia y desarrollo de tecnologías bajas en carbono, particularmente en la administración pública centralizada y de los municipios.

Para lograr avanzar en los elementos que integran la gestión climática, se establecería como instrumentos de planeación y gestión de: La Estrategia Nacional de Adaptación y Mitigación al cambio climático (que estaría incorporada en el PNDH); el Plan Nacional de Adaptación y Mitigación; el Programa Nacional de Innovación Tecnológica por sectores; las Comunicaciones Nacionales junto al Inventario Nacional que sea más actualizado, la Línea de Base sectorial y los Informes de Evaluación Bianual, el Informe Nacional de cambio de uso del suelo por el sector agrícola y forestal que se 
elaboren, como instrumento de evaluación anual de la gestión climática y la huella de carbono.

La presente propuesta de Ley gozaría de un reglamento, el cual debería ser presentado por el MARENA en un plazo determinado para aprobación del poder ejecutivo, siendo luego publicado en La Gaceta para entrar en vigor en todo el territorio nacional.

\section{vil. Conclusiones}

Al concluir encontramos que, los Derechos Humanos fundamentales de las personas han adquirido en los últimos años una importancia trascendental nacional e internacionalmente, que se observa con la incorporación en los ordenamientos jurídicos de los Estados del actual sistema internacional, en las políticas de los gobiernos y en el marco jurídico de todos los Estados.

Que en el desarrollo histórico de los Derechos Humanos, clasificados en etapas, vemos que la tercera generación incluye los derechos a la protección del patrimonio cultural, al derecho al desarrollo, al derecho a un medio ambiente adecuado que incluye el sistema climático, a la protección al planeta como hábitat único de la especie, el derecho a la asistencia humanitaria, al manejo, uso y protección de los recursos naturales y del concepto de la solidaridad; donde encontramos integrado el sistema climático incorporado, debido a que éste integra un conjunto de elementos sociales, atmosféricos, productivos, económicos, culturales, bióticos y no bióticos (entre los más importantes), que interactúan entre sí con el ser humano en un espacio y tiempo determinado para la realización de todos los derechos del individuo, en su medio y contexto, siendo éste una proyección del derecho a la vida y a un equilibrio ecológico de preservación de la existencia en el planeta.

Visto así, el derecho humano a vivir y a disfrutar de un sistema climático en equilibrio encuentra su razón de ser, como un derecho individual, colectivo y universal, con características que pueden 
emanar de los derechos civiles y políticos, como también con otros que, provienen de los derechos económicos, sociales y culturales, para luego transitar, hacia los denominados derechos de solidaridad y del medio ambiente, siendo a la vez la atmósfera el bien jurídico ambiental considerado como parte inherente del concepto ambiental indeterminado, multifacético y diverso, pues por su propia naturaleza, el clima no está determinado por una delimitación imaginaria que otorga soberanía absoluta a determinada nación, sino que éste tiene un carácter global, así como por las funciones que este realiza para todos los individuos para disfrute de los Derechos Humanos como tal.

Resulta inconcebible disfrutar el pleno goce de los Derechos $\mathrm{Hu}-$ manos sin un sistema climático sustentable, el cual preserve condiciones propicias para el desarrollo humano sostenible, ante la magnitud y alcances de los efectos adversos del problema climático. De esa manera, el bien jurídico a proteger es el sistema climático global, él que debe ser preservado por el Derecho Internacional del Medio Ambiente para las generaciones actuales y futuras, puesto resulta una fusión de los conceptos de interés común y de responsabilidad ética para la generación futura, la que parte de una obligación ineludible de los Estados.

De ahí que no es una mera expresión mencionar que, los países en desarrollo son las naciones más vulnerables, donde el sistema socioeconómico y sus instituciones son menos favorables para enfrentar la vulnerabilidad frente al cambio climático, por la pobreza y la desigualdad o el acceso a fuentes de financiamiento, pues se ven limitadas su capacidad adaptativa, por contar con escasa disponibilidad de medios tecnológicos y recursos financieros para implementación de medidas en la adaptación, a como se demuestra con el caso nicaragüense.

En ese contexto, encontramos que Nicaragua genera una emisión pércapita de gases de efecto invernadero tan baja con apenas el $0,03 \%$, pero que ha sido afectada y tendrá consecuencias funestas 
en su desarrollo, por las implicaciones negativas del calentamiento global sin la responsabilidad histórica de la causalidad climática.

De forma complementaria, se ha considerado a Nicaragua como país multiamenazas, tanto por los riesgos geológicos y sísmicos que se complementan con los eventos extremos hidrometeorológicos inducidos por el cambio climático, que exacerba la poca capacidad adaptativa e incrementa la vulnerabilidad frente a otros tipos de presiones, como la pobreza en el país.

Sin embargo, la gestión climática no cuenta con un instrumento normativo en esta materia de forma exclusiva, pues la temática se ha visto como un tema transversal y no como un objeto para preparar las condiciones que faciliten su mitigación, así como adaptarnos a las consecuencias, lo que al considerar a Nicaragua como país de alto nivel de vulnerabilidad y del riesgo; que ha sido y será impactado por el cambio climático con mayor rudeza; posee cierta dispersión legal y normativa en determinados instrumentos de política pública.

Se considera oportuno sugerir una propuesta de ley que fortalezca la capacidad adaptativa para el manejo de la problemática ambiental y del sistema climático, que facilite la resiliencia climática, los servicios ambientales, la mitigación y la adaptación por el otro, frente a los escenarios que el país enfrenta a futuro próximo.

\section{Vili. Fuentes consultadas}

\section{Bibliografía}

Garcia, Emilio, Derechos humanos y calidad de vida, Madrid, Universidad Complutense, 1999.

Jaguenod de Zsogon, Silvia, Iniciación al derecho ambiental, España, Dikinson, 1996.

Juste Ruiz, José, La Protección del Medio Ambiente en su Dimensión Internacional, Madrid, Mcgraw-Hill/Interamericana de España, 1999. 
Martín, Mateos, Tratado de Derecho Ambiental, Vol. I, Madrid, Trívium, 1991.

Pagiola, Stefano, Bishop, Joshua y Landell-Mills, Natasha, Selling Forest Environmental Services, Market-Based Mechanisms For Conservation And Development, Londres, Earthscan Publications, 2002.

Risse, Thomas, et al., The Power of Human Rights: International Norms and Domestic Change, New York, Cambridge University Press, 1999.

Wunder, Sven, Pagos por servicios ambientales: principios básicos esenciales, Indonesia, Cifor, 2006.

\section{Hemerografía}

BCN, Nicaragua En Cifras, Managua, Nicaragua, 2013. Http://Wwre. Bcn.Gob.Ni/Publicaciones/Periodicidad/Anual/Nicaragua_Cifras/2013/Nicaragua_Cifras.Pdf.

Índice mensual de actividad económica, Managua, Nicaragua, 2014. Http://Wwrw.Bcn.Gob.Ni/Publicaciones/Periodicidad/Mensual/Imae/0914/Informe.Pdf.

BID, Vulnerabilidad y adaptación al cambio climático: diagnóstico inicial, avances, vacios y potenciales líneas de acción en Mesoamérica, Washington, USA, 2010. Https://Publications.Iadb.Org/Handle $/ 11319 / 1761$ ? Locale-Attribute $=$ En .

Banco Interamericano de Desarrollo / Banco Mundial, Perfil del riesgo de desastres de Nicaragua, Washington, USA, 2010. Https://Publications.Iadb.Org/Bitstream/Handle/11319/6973/Indicadores_Riesgo_Desastre_Gestion_Riesgos_Programa_America_Latina_Caribe_Nicaragua.Pdf. 
BID, Indicadores de Riesgo De Desastre y de Gestión de Riesgos: Programa para América Latina y el Caribe: Nicaragua, Washington, USA, 2015. Https://Publications.Iadb.Org/Handle/11319/6973?Locale-Attribute $=$ Es.

BM, Nicaragua: informe sobre la pobreza 1993-2005, Washington, USA, 2008 Https://Bjcu.Wordpress.Com/Tag/Banco-Mundial/.

Boyle, Allan, "Human rights or environmental rights? a reassessment", Fordham Environmental Law Review, Vol. XVIII, 2007. Http://Www.Law.Ed.Ac.Uk/Includes/Remote_People_Profile/ Remote_Staff_Profile?Sq_Content_Src $=\% 2 b d x j s p w h 0 d h a l m 0 e l m-$ kylmkz3d3cylmxhdy 5 lzc 5 hyy 51 ayuyrmzpbgvfzg 93 bmxvywqlmkzwdwjsawnhdglvbnmlmkywxzeymiffahotyw 5 yawdodhnvcmvudmlyb25tzw50ywxyawdodhnhcmohc3nlcy 5wzgymywxspte\%3d.

Chifford, Bob, "New Human Rights Issues: Understanding Their Contentious Rise", Scielo, 2009. Http://Wwrw.Scielo.Org. Co/Scielo.Php?Script=Sci_ArttextEPid=S0121-56122009000100002

Cecchetto, Sergio, “'Una ética de cara al futuro? Derechos humanos y responsabilidades de la generación presente frente a las generaciones por venir", Andamios, Revista de Investigación Social, Vol. 3, Núm. 6, 2007. Http://Wwrw.Redalyc.Org/Articulo. Oa? Id $=62830603$

Germanwatch, "Who Suffers Most from Extreme Weather Events? Weather-Related Loss Events In 2012 And 1993 To 2012", Global Climate Risk Index 2014, 2013. Http://Germanwatch.Org/ De/Download/8551.Pdf

"Who Suffers Most from Extreme Weather Events? Weather-Related Loss Events In 2013 And 1994 To 2013", Global Climate Risk Index 2014, 2014. Http://Germanwatch.Org/ En/Cri 
INIDE, Encuesta de demografia y salud - 2011 y 2006/2007, Managua, Nicaragua, 2011. Http://Inide.Gob.Ni/Bibliovirtual/Publicaciones.Htm.

Jiménez Solares, Elba, "Las normas internacionales de derechos humanos y su contribución al orden público internacional”, Revista de Derecho, Núm. 14, México, Universidad Nacional Autónoma de México, 2014, pp. 325-347. Https://Dialnet.Unirioja.Es/Servlet/Articulo? Codigo $=4965197$

Juste Ruiz, José, "El desarrollo sostenible y los derechos humanos", en Curtis, Cristian, Hauser, Denise y Rodriguez Huerta, Gabriela (Coords), Revista de Derechos Humanos, México, PorrúaITAM, 2005. Https://Revistas.Furidicas.Unam.Mx/Index.Php/ Derecho-Internacional/Article/View/174/285.

Loperena Rota, Demetrio, "La posición jurídica del ciudadano ante el medio ambiente", Revista Naturzale, No. 12, 2007. Http:// Wwr.Euskomedia.Org/Pdfanlt/Naturzale/12/12057073.Pdf

Marena, Informe del estado ambiental en Nicaragua, Managua, 2001. Http://Wwre.Pnuma.Org/Deat1/Pdf/Geonicaragua2001.Pdf.

, Segunda comunicación nacional ante la convención marco de las Naciones Unidas sobre cambio climático, Managua, 2008. Http:// Unfccc.Int/National_Reports/Non-Annex_I_Natcom/Submitted_ Natcom/Items/653.Php.

PNUD, Informe sobre desarrollo humano 2014 - resumen -; sostener el progreso humano: reducir vulnerabilidades y construir resiliencia, New York, 2014. Http://Www.Undp.Org/Content/Undp/Es/Home/ Librarypage/Hdr/2014-Human-Development-Report/ . 
3. Legisgrafía

Constitución Política de Nicaragua. Http://Www.Poderjudicial.Gob. Ni/Pjupload/Archivos/Documentos/La_Constitucion_Politica_ $Y_{-}$ Sus_Reformas(3).Pdf.

Ley No. 217, Ley general del medio ambiente y los recursos naturales, publicada en la Gaceta no. 105, Nicaragua, 6 de junio de 1996.

Ley No 462, Ley de conservación, fomento y desarrollo sostenible del sector forestal, publicada en la Gaceta no. 168, 4 de septiembre del 2003.

Ley No. 620, Ley de aguas nacionales, publicada en la Gaceta no. 169, 4 de septiembre del 2007. 
